\title{
Spotter: A Model Based Active Geolocation Service
}

\author{
Sándor Laki*, Péter Mátray ${ }^{\dagger}$, Péter Hága $^{\dagger}$, Tamás Sebők ${ }^{\dagger}$, István Csabai ${ }^{\dagger}$ and Gábor Vattay ${ }^{\dagger}$ \\ ${ }^{*}$ Department of Information Systems, ${ }^{\dagger}$ Department of Physics of Complex Systems \\ Eötvös Loránd University, Budapest, Hungary \\ E-mail: \{laki, matray, haga, sebok, csabai, vattay\}@ complex.elte.hu
}

\begin{abstract}
The localization of Internet hosts opens space for a wide scope of applications, from targeted, location aware content provision to localizing illegal content. In this paper we present a novel probabilistic approach, called Spotter, for estimating the geographic position of Internet devices with remarkable precision. While the existing methods use landmark specific calibration for building their internal models we show that the delay-distance data follow a generic distribution for each landmark. Hence, instead of describing the delay-distance space in a landmark specific manner our proposed method handles all the calibration points together and derives a common delay-distance model. This fundamental discovery indicates that, in contrast to prior techniques, Spotter is less prone to measurement errors and other anomalies such as indirect routing. To demonstrate the robustness and the accuracy of Spotter we test the performance on PlanetLab nodes as well as on a more realistic reference set collected by CAIDA explicitly for geolocation comparison purposes. To the best of our knowledge, we are the first to use this novel ground truth containing over 23000 network routers with their geographic locations.
\end{abstract}

\section{INTRODUCTION}

Since the last decade many location aware applications have been appearing from content localization to targeted advertisements. Furthermore most of the online services could benefit from determining the geographic locations of their users. Besides the commercial utilization, the IP localization became important in network research and development as well, e.g. to visualize the results of Internet measurements, investigate the geographic aspects of various important problems like network topology, policy routing and pricing strategies. In addition, it opens space for a wider scope of applications, from targeted, location aware content provision to fraud detection.

Nevertheless, determining geographical location of Internet hosts by a single IP address poses many challenges, since there is no direct relationship between the IP address of a network device and its geographic location. Most of the existing geolocation services are based on databases which store organizational information assigned to IP domains, or try to infer location information from DNS names. Usually the accuracy of these services is insufficient due to the lack of reliable information. To overcome the uncertainty of these registry based approaches, active geolocation techniques have emerged [1]-[3]. These methods attempt to approximate the geographic distance based on delay and topology measurements and then use triangulation-like methods to obtain the geographical location of Internet hosts. Most of them introduce strict geographical constraints which determine a region in which the target should be located with equal probabilities.
This paper presents a probabilistic geolocation approach, called Spotter, which based on the detailed statistical analysis of the relationship between network delay and geographic distance. This analysis reveals that the distribution of spatial distances for a given delay follows a common distribution and is independent of the landmark's position from where the measurement was performed. While the state-of-the-art techniques use separate calibration data for each landmark to determine their internal models, our method handles all these data together to derive a generic delay-distance model. By exploiting the benefits of this fundamental discovery our novel probabilistic approach is less prone to measurement errors and other network anomalies affecting the distance approximation. In addition, for a given target Spotter returns not only the estimated coordinates, but also a spatial probability surface describing how likely the target is at given regions of the globe. To demonstrate the robustness and the accuracy of Spotter we test the performance on PlanetLab nodes as well as on a more realistic reference set collected by CAIDA [4] for the purposes of a geolocation comparison survey. The latest set contains more than 23000 distinct network interfaces with reliable location information. Our performance analysis shows that on both ground truth data sets Spotter provides location estimations with outstanding accuracy compared to the nonprobabilistic models of the state-of-the-art techniques, CBG [2] and Octant [3].

To manifest the applicability of our probabilistic approach we developed a publicly available geolocation service ${ }^{1}$ [5]. In contrast to the prior works, our solution is based on a fundamentally different principle and combines the benefits of active measurements by applying a novel probabilistic model for presenting a reliable and accurate geolocation service.

The rest of the paper is organized as follows: in Section II we briefly overview the available geolocation approaches from commercial services to advanced active geolocation techniques. Section III delineates the theoretical background of our probabilistic geolocation methodology. In Section IV we present a detailed statistical analysis of the relationship between network delay and geographic distance and show the generality of the delay-distance distribution. Besides this, we examine the accuracy of location estimations using two different reference data sets as geolocation ground truth. The online geolocation service built upon our probabilistic model is briefly introduced in Section V. To demonstrate the power

\footnotetext{
${ }^{1}$ The SPOTTER website: http://spotter.etomic.org
} 
of Spotter we outline some large scale localization scenarios in Section VI, while the final section summarizes our results.

\section{StATE of ThE ART}

During the recent years several geolocation techniques have emerged, all of them aim to give an accurate approximation of the location of network hosts which are not known a priori. The different approaches can be classified into two groups depending on their underlying methodology to collect location information. One set of methods uses previously registered data to gain information on the geographic location of IP addresses. We refer to these as registry based approaches. Other techniques utilize active delay and topology measurements to approximate IP locations. We call them measurement based approaches.

\section{A. Registry Based Geolocation}

Many of the existing geolocation techniques use Whois databases, DNS LOC records or DNS names [6] to determine the location of a given host. From Whois databases one can retrieve the name and street address of the organization which registered the address block. However, for a large ISP or a geographically dispersed organization the registered street address usually differs from the real location of its hosts. A similar problem arises in the use of DNS names, since the names can be both useful or misleading due to the naming conventions of the ISP [7]. Other registry based techniques include commercial approaches, e.g. the gathering of user submitted location data from commercial websites, or network reconnaissance, where one obtains the description of the geographic layout of an ISP's network and internal routing policies.

In general we can say that registry based methods can provide very accurate location approximations, however in many cases their errors are very large for entire blocks of IP addresses [8]. This feature is due to the fact that their precision greatly depends on the resolution and reliability of the previously registered data they use.

To illustrate the problem we have queried whois.arin.net to collect locations for IP addresses assigned to Google Inc. According to the Whois query, $99.7 \%$ of the IPs are located in Mountain View, California, where Google's headquarters can be found. As Table I shows, only 537 IP addresses out of the total 222877 are located in 12 other cities. To investigate the reliability of this result we have selected 4000 addresses from the Google domain and measured delays to them from the PlanetLab node at Universidad Publica de Navarra, Pamplona, Spain. Assuming an ideal case where the signal propagation speed is $200000 \mathrm{~km} / \mathrm{sec}$, for all the 4000 IPs we can determine the maximal physically possible distance from Pamplona. In Figure 1 we present the cumulative distribution of these maximal distances and also indicate the distance between Pamplona and Mounatin View. The plot shows that $47 \%$ of the examined hosts have maximal distance below the real Pamplona-Mounatin View distance and therefore, in principle these nodes cannot reside in Mountain View.
Table I

LOCATIONS OF NORTH AMERICAN IPV4 ADDRESSES ASSIGNED TO GOOGLE INC.

\begin{tabular}{lc} 
Registered location & number of distinct IPv4 address \\
\hline \hline Mountain View, CA, US & 222340 \\
Plano, TX, US & 331 \\
Chicago, IL, US & 46 \\
Irvine, CA, US & 30 \\
Waterloo, ON, CA & 22 \\
San Francisco, CA, US & 21 \\
Atlanta, GA, US & 15 \\
Phoenix, AZ, US & 15 \\
Southfield, MI, US & 15 \\
Mequon, WI, US & 14 \\
Pittsburgh, PA, US & 14 \\
Gladwyne, PA, US & 7 \\
Richardson, TX, US & 7 \\
\hline Total & 222877 \\
\hline
\end{tabular}

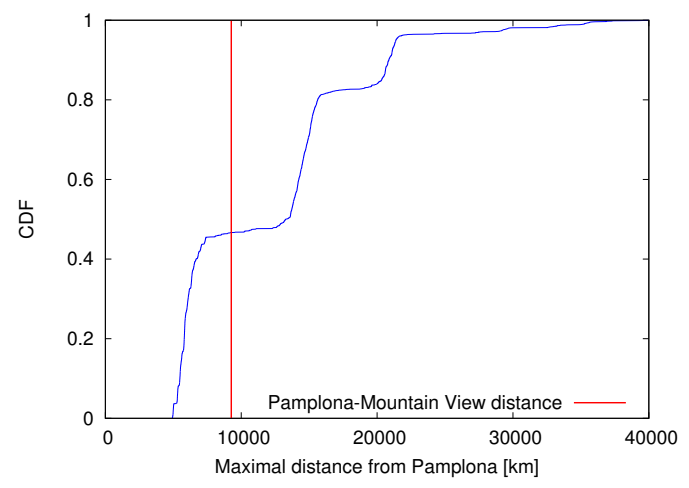

Figure 1. Cumulative distribution of the maximal distances from Pamplona, Spain to 4000 Google IPs. The maximal distances are calculated from the network delays assuming $200000 \mathrm{~km} / \mathrm{sec}$ signal propagation speed. The vertical line represents the real geographical distance between Pamplona and Mountain View, CA, showing that $47 \%$ of the nodes must be closer to Pamplona than Mountain View.

\section{B. Measurement Based Geolocation}

Some other proposals apply a different approach and utilize active measurements to overcome the above limitations. IP2Geo [6] contains a tool called Geoping, which tries to approximate the geographical location of network hosts on the basis of packet delay measurements. A more mature approach is the simultaneous application of several delay constraints to infer the location of a network host. This is done by constraint-based-geolocation (CBG) techniques [2]. CBG introduces a triangulation-like method to combine the distance estimates from all landmarks. To estimate delaydistance relation, each landmark measures the delay from itself to all the others. From these inter-landmark measurements $\mathrm{CBG}$ can be calibrated by data fitting [2]. In general, each delay measurement defines a circle around the landmark from where the delay was measured. The possible locations of the target node are determined by intersecting all of these circles. In most 
of the cases this intersection produces a region in which the target node must be located. In [1] the authors extend the CBG with novel end-to-end path constraints and apply a detailed latency model for the better approximation of geographic distances. By this the localization accuracy is significantly improved for intermediate routers, but for arbitrary network hosts the applicability is limited.

Another technique is where the topology information and latency measurements are used together in the location estimation. This method type is called topology based geolocation (TBG) [9]. TBG localizes all the intermediate routers between the landmarks and the target node. This approach is based on link-latency estimations and on precise topology discovery. The basic tools of this method are traceroute and interface clustering applications. Later works such as Octant [3] enhance the accuracy of location approximations by combining TBG with various other techniques, including DNS and whois lookups or clipping regions with negative geographic and demographic constraints.

\section{Geolocation Services}

As IP geolocation gained importance in the last decade, various services have emerged to provide such information. To answer IP location queries nearly all of today's well known geolocation services ${ }^{2}$ maintain a repository that stores previously collected geodata. Most providers use registry based methods to build up their location database. Naturally, many providers apply further refinements on raw registry based data, and tend to use a wider variety of data sources. For instance, MaxMind [11] and Digital Envoy Netacuity [12] collect geographic information from partner websites where users enter their location [10], or Quova [13] purchases internal routing data directly from ISPs [14].

Although larger providers apply different validation techniques, the error patterns of the base registry data cannot be fully eliminated. To demonstrate the imperfection of the data cleaning algorithms, we have localized five randomly selected GÉANT routers with known positions spread across Europe using MaxMind. We found that MaxMind located all of the routers in Cambridge, UK, where the GÉANT operator is registered. This mislocation indicates that MaxMind relies on previously registered Whois data, which leads to unreliable results in this case. A recent study of geolocation databases [10] shows that similar anomalies are present in other well known geolocation services as well.

Measurement based geolocation tools mostly emerge from universities and research institutes. Usually these tools do not provide a service interface to query IP addresses at all, and even if they do so, it is usually suitable for demonstration purposes only. The absence of reliable measurement based geolocation services sets back many research and application scenarios where the geographic position of an Internet host has a significant role. In the following we introduce a novel probabilistic geolocation model and a service built on top of that to fill this gap.

\footnotetext{
${ }^{2}$ See [10] for a list of popular services.
}

\section{THEORETICAL BACKGROUND}

In the typical scenario of measurement based geolocation we have landmark nodes with known geographic location and a target node without known position. To approximate the location of the target we measure propagation delays from the landmarks to the target, and then convert the delays into geographic distances based on a delay-distance model. The resulting set of distance constraints is used to determine the target's estimated location with a triangulation-like method. The heart of this process is the delay-distance model, which we introduce in detail in the following.

\section{A. Probabilistic Delay-Distance Model}

We developed a probabilistic model to determine the most likely position of the target based on signal propagation delay values between the landmarks and the target. To approach the problem, we first examine the case where we have only one landmark. Let $L$ denote the landmark given by its latitude and longitude coordinates:

$$
\mathbf{L}=\left(L_{\text {lat }}, L_{\text {lng }}\right) .
$$

Let $T$ represent the target node, whose actual $\mathbf{T}=\left(T_{\text {lat }}, T_{\operatorname{lng}}\right)$ coordinates are unknown. ${ }^{3}$ In the following we describe the target's position with a random variable $\tau$ :

$$
\tau=\left(\tau_{\text {lat }}, \tau_{\text {lng }}\right) .
$$

The spatial probability density function of $\tau$ determines how likely the target is at given regions of the globe. In our approach the density function depends on the location of the landmark and the signal propagation delay between $L$ and $T$. For a given $\mathbf{L}$ and a fixed delay $d$ we denote this function by $g_{d}^{L}(\tau)$. With this notation the conditional probability that $\mathbf{T}$ falls into region $H$ is provided by

$$
P(\mathbf{T} \in H \mid L \bowtie d)=\int_{H} g_{d}^{L}(\tau) \mathrm{d} \tau,
$$

where the condition $L \bowtie d$ indicates that the propagation delay between $L$ and $T$ is $d$. As delay measurements do not carry any information on the bearing from $L$ to $T$, we can derive constraints only on their distance. Hence, in our mathematical construction we assume an isotropic behavior where the distance probabilities are equal in all geographic directions from $L$, for a given $d$. Hence, $g_{d}^{L}$ defines a ring-like surface around the landmark.

\section{B. Location Approximation}

Due to the isotropic nature of (1) a single probability surface does not provide a well-defined location for the target. To enable definite estimations we extend the construction for multiple landmarks and consider the resulting spatial density distributions jointly to maximize the probability of the location.

${ }^{3}$ Note that while $L$ and $T$ represent the nodes themselves, the boldface $\mathbf{L}$ and $\mathbf{T}$ stand for their coordinates. 


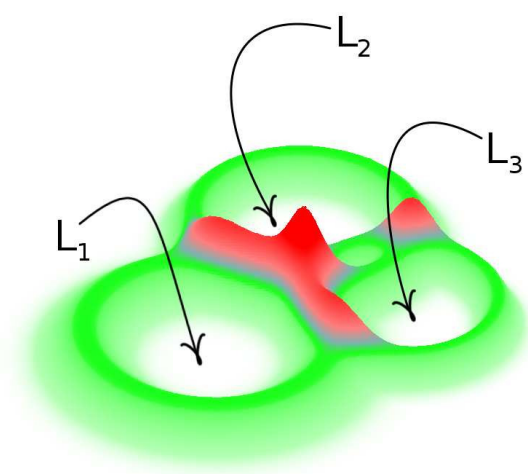

(a) Probability density surfaces

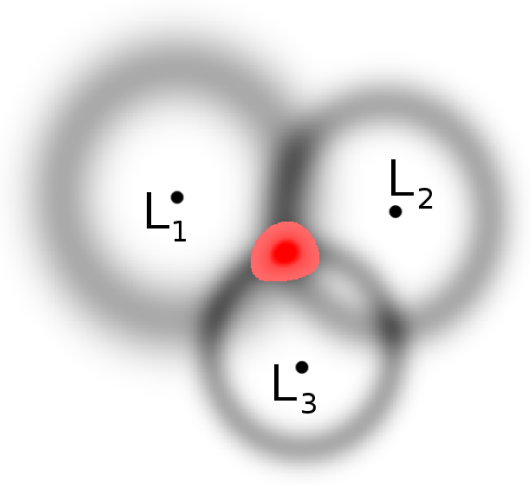

(b) Estimated region

Figure 2. Target localization from three landmarks. (a) The sum of the probability density surfaces for three landmarks illustrates the ring-like structure of the individual spatial densities. The locations denoted by green color are less probable than the red ones where the three rings meet. (b) The previous three density functions are shown in gray scale. The joint probability surface calculated by (2) are displayed in pink, while the region with red color illustrates the estimated region for a given confidence level.

Let us suppose we have $n$ landmarks: $L_{1}, \ldots, L_{n}$. Let $d_{i}$ denote the propagation delay from $L_{i}$ to $T$ and $g_{d_{i}}^{L_{i}}(\tau)$ represent the corresponding spatial probability densities. The conditional probability that the target is located in a certain $H$ region can be expressed as the product of the distinct densities:

$$
\begin{aligned}
P(\mathbf{T} & \left.\in H \mid L_{1} \bowtie d_{1}, \ldots, L_{n} \bowtie d_{n}\right)= \\
& =A_{H} \prod_{i=1}^{n} P\left(\mathbf{T} \in H \mid L_{i} \bowtie d_{i}\right)= \\
& =A_{H} \prod_{i=1}^{n} \int_{H} g_{d_{i}}^{L_{i}}(\tau) \mathrm{d} \tau .
\end{aligned}
$$

Here $A_{H}=P(\mathbf{T} \in H)^{1-n}$ where $P(\mathbf{T} \in H)$ stands for the unconditional probability that $\mathbf{T}$ resides in $H$. This expression presumes that the delays measured from different landmarks are statistically independent. Although this condition is not automatically ensured, the geographically dispersed distribution of landmarks and a careful landmark selection method together reduce the dependence of the measurements. If we have prior assumptions on the possible locations of the target, then the distribution of $P(\mathbf{T} \in H)$ might convey this information. For instance, population density data or various geographic constraints can be coded into $P(\mathbf{T} \in H)$. In the following we do not apply such assumptions, but rather suppose that $P(\mathbf{T} \in H)$ is uniformly distributed. Hence, $A_{H}$ solely depends on the area of $H$ and can be substituted with a constant for all regions having the same area.

The basic principle of our localization method is that the estimated position must fall into the region designated by the $g_{d_{i}}^{L_{i}}$ functions such that the joint probability in (2) is maximized for a reasonably sized region $H$. To illustrate the concept in Figure 2(a) we plotted the $g_{d_{i}}^{L_{i}}$ probability densities for three landmark nodes. For the sake of demonstration the joint region of the rings are visualized by the sum of the distinct probability values instead of applying the product in (2). On the contrary, Figure 2(b) shows the valid calculation according to (2) for the same densities.

To determine the spatial probability density of $\tau$ we divide the surface of the globe into a finite number of cells with equal size and calculate (2) over them (see Section V-A for details). This way we obtain a probability value for each cell, which is a much finer information on the possible position of the target than previous localization methods could provide. To deliver position estimations from the individual cell probabilities we can apply one of the following methods.

In case an estimated region of the target is needed, we can define the union of the most probable cells according to a required confidence level. The estimated region for two different confidence levels are indicated with colors in Figure 2(b). One can observe that the regions emerge where all the involved rings provide high probability values. Estimating the target location with a region might be useful in the visualization of results of large scale host localization (see Section VI for examples).

On the other hand, for certain purposes an estimated region is not appropriate, instead a single "best" target location value is necessary. Due to the nature of our probabilistic approach there are several choices to define a single location. For instance, one can simply select the center of the estimated region or use the coordinates corresponding to the maximum or mean value of the probability distribution.

\section{Determining the Spatial Density Functions}

To be able to apply the previous theoretical results we need to infer the $g_{d}^{L}$ probability densities. For this we make two natural assumptions. First, we suppose that $g_{d}^{L}$ is isotropic, since we have no reason to doubt that signal propagation characteristics agree in different geographical directions. This hypothesis implicitly appears in most of the previous geolocation works, including [2], [3], [9], [15]. As a consequence, we can describe $g_{d}^{L}$ with its $f_{d}^{L}$ radial profile. Essentially, $f_{d}^{L}$ is a one dimensional distance distribution for $L$ and $d$. 
Second, in contrast to prior works of Wong et al. [3] or Eriksson et al. [15] we expect that the $f_{d}^{L}$ distribution is independent of the actual location of $L$, and thus it can be substituted with an $f_{d}$ distance distribution common for all landmarks. This fundamental step will be discussed later in the section, since it has a serious impact on the quality of calibration.

\section{A. Approximating the Radial Profile}

To benefit from the previous two assumptions, instead of determining $g_{d}^{L}$ itself, we approximate the $f_{d}$ common distance distribution. This is done by a thorough analysis of real world calibration data collected in PlanetLab. Figure 3(a) presents the gathered 40000 data points. Each dot represents the measured round-trip delay value and the distance between a PlanetLab node pair. The great circle distance is calculated from the known coordinates of the PlanetLab nodes.

To understand the nature of the calibration data set we determined the probability distribution of the standardized data values. As presented in Figure 3(b) there is a good match with the standard normal distribution (the fitting parameters are $\mu=-0.078, \sigma=1.035)$. This feature indicates that $f_{d}$ is well approximated by a normal distribution at any fixed $d$ delay. To characterize the mean and variance of $f_{d}$, we fitted the $\mu(d)$ and $\sigma(d)$ polynomials to the data set. These curves are also shown in Figure 3(a). The above observations enable a simple but consistent approximation of $f_{d}$ with a normal distribution:

$$
f_{d}(s) \approx \frac{1}{\sqrt{2 \pi \cdot} \sigma(d)} \cdot \exp \left(-\frac{(s-\mu(d))^{2}}{2 \sigma^{2}(d)}\right),
$$

where $s$ is a random variable describing the distance. During the evaluation process the (3) distance distribution is used to calculate the $g_{d}^{L}(\tau)$ spatial probability densities. For a given $L \bowtie d$ this is done by the following formula:

$$
g_{d}^{L}(\tau)=A_{d} \cdot f_{d}(S(L, \tau)) .
$$

Here, $S(L, \tau)$ represents the great-circle distance between $L$ and $\tau$, while $A_{d}$ denotes the normalization factor.

\section{B. The Generality of the Delay-Distance Distribution}

Now, we revisit our prior assumption and argue that the individual landmarks have a generic underlying delay-distance behavior. To investigate the validity of the hypothesis we check if delay-distance data corresponding to individual landmarks follow the distribution of the overall data set presented in Figure 3(a). This test is done by means of Q-Q plot. According to the standard notations the standardized values of the landmark specific data are called observed values, while we refer to the standardized values of the overall data set as expected values. In Figure 3(c) we present the Q-Q plot where the corresponding quantiles of the observed and the expected values are plotted against each other. For a better visibility, only the data for five selected landmarks are shown which are considered to represent the whole landmark set well. The good match with the diagonal indicates that the expected and the observed values follow the same delay-distance distribution. Consequently, the soundness of our original assumption is verified.

This conclusion gains importance during the empirical calibration of delay-distance models. Currently, some hundreds of ground truth locations are publicly available, most of which belong to PlanetLab. The number of reachable ground truth positions determines the size of the calibration data set. To infer landmark specific delay models the overall calibration set has to be divided into significantly smaller landmark specific chunks. These smaller data sets contain only a few hundred points (as many as the number of known ground truth positions). Due to the small sample size it is technically infeasible to infer reliable landmark specific delay models. Accordingly, geolocation approaches using landmark specific calibration might suffer from inconsistent location approximation.

As a novelty, we use the overall calibration set to determine a common delay-distance model for all the landmarks. In contrast to landmark specific calibration this can be done with sufficient accuracy and resolution which eventually provides consistent geographic constraints across the landmarks.

\section{Performance Analysis}

We examine the localization accuracy of our approach and compare the results with the performance of the state-ofthe-art delay models of $\mathrm{CBG}$ and Octant. As discussed in Section II CBG determines a flat disk around each landmark in which the target node should be located. To derive the disk around a landmark CBG uses the tightest line fit above delaydistance pairs. For more details see [2]. In contrast to $\mathrm{CBG}$, Octant generates flat rings around the landmarks to describe the possible locations of the target [3]. To this end, Octant combines passive and active techniques in order to define positive and negative geographical constraints.

To enable performance comparison we have reimplemented the delay models behind $\mathrm{CBG}$ and Octant according to [2] and [3]. Since we focus solely on the performance of delay models we did not reproduce all the features of the original frameworks.

To examine the accuracy of the three geolocalization methods we use two different reference data sets as geolocation ground truth. First we apply the methods on the PLANETLAB set containing the PlanetLab nodes as reference targets, since their geographical positions are well known. This reference set is widely used in the geolocation literature both for calibration and validation purposes. We follow the common verification scenario where every PlanetLab node is selected as a target and then geolocalized using all the remaining nodes as landmarks.

To determine the localization errors we calculate the greatcircle distance between the estimated and the actual coordinates. The upper row of Figure 4 shows the cumulative distribution of the localization errors of Spotter, CBG and Octant models on these targets.

For all regions $13 \%, 10 \%$ and $3 \%$ of the hosts have less then $10 \mathrm{~km}$ error with Spotter, Octant and CBG, respectively. The $50 \mathrm{~km}$ range includes the $35 \%, 26 \%$ and $23 \%$ of the 


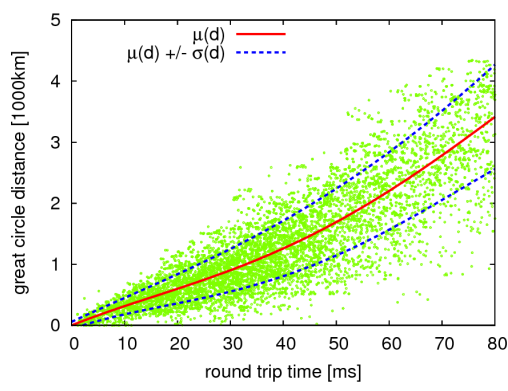

(a) Delay-distance plot, where each dot represents a measurement between two PlanetLab nodes. The fitted $\mu(d)$ mean and $\sigma(d)$ standard deviation curves are shown.

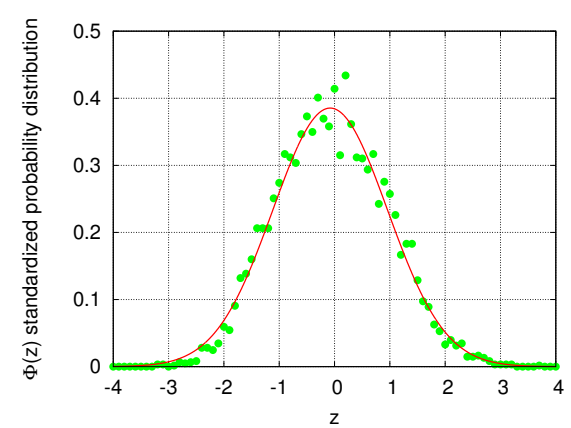

(b) The probability distribution of the standardized delay-distance data indicates generic behavior.

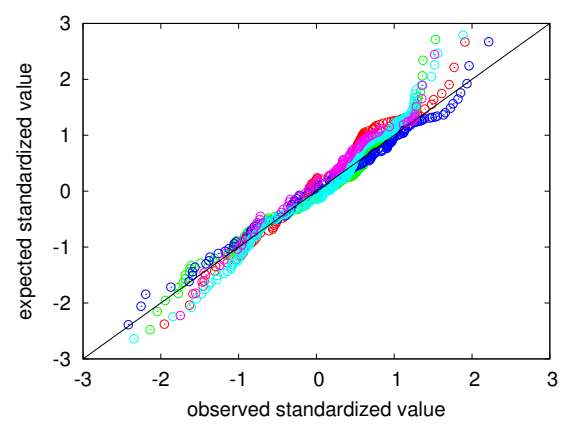

(c) Q-Q plot for five selected landmarks shows that the individual landmarks follow the same normal distribution.

Figure 3. Fundamental observations on the delay data collected between the PlanetLab node pairs.

hosts. The median errors produced by the methods on the full set are 75,125 and $175 \mathrm{~km}$. These numbers show that the probabilistic approach outperforms the flat models. Nevertheless, the applicability of this verification scenario is limited, since all the models are validated on the very same environment as they were calibrated on previously.

To investigate the methods in a more realistic scenario we use a more representative data set collected by CAIDA [4] for the purpose of a geolocation comparison survey. This set is independent of the geolocation models in the sense that it was not involved in their calibration process. This data set contains more than 23000 distinct IP addresses geographically distributed across North America and Europe. These IPs belong to network routers of Cogent, a large Tier-1 ISP. We refer to this data set as COGENT.

The cumulative distribution of localization errors on the COGENT set are presented in the lower row of Figure 4. One can observe that Spotter has outstanding accuracy compared to Octant and CBG. $35 \%$ of the North American nodes are localized within $10 \mathrm{~km}$ error, while Octant and CBG have only $2 \%$ and $9 \%$ of the estimates within the same range. For our approach almost $70 \%$ of the targets have error less than $50 \mathrm{~km}$, while Octant and CBG estimate $27 \%$ and $40 \%$ of the nodes in the same range. The accuracy on the European targets decreases for all the methods. Spotter localizes $19 \%$ of the hosts within $10 \mathrm{~km}$, while in case of the other two methods only $1 \%$ of targets have error within this range. The $50 \mathrm{~km}$ range contains $40 \%, 4 \%$ and $8 \%$ of the nodes in the same order. It can also be seen that on the full COGENT data set the median errors are 30, 120 and $100 \mathrm{~km}$ for Spotter, Octant and $\mathrm{CBG}$, respectively.

Examining the European results on COGENT in more depth we found that the measurements are highly affected by indirect routing that has negative effect on distance estimations. According to our analysis Spotter handles this phenomenon better than CBG and Octant. This is due to the fact that the latter models produce strict constraints around the landmarks, while Spotter's underlying probabilistic approach is less prone to measurement errors.
Comparing the numerical results Spotter shows significantly better performance on both reference data sets and appears to be more robust against measurement anomalies. According to a recent analysis of Shavitt et al., [10] shows that Spotter's accuracy is comparable to most of the well-known registry based geolocation services.

\section{THE SERVICE}

In the previous sections we introduced a statistical approach for localizing Internet hosts. Although this can be viewed as the heart of the geolocalization process, for a reliable service we need to implement several additional modules ranging from data collection to result visualization. Next, we introduce the Spotter service which integrates these diverse tasks into a complete solution.

\section{A. Localization Workflow}

In order to enable easy extensibility the Spotter implementation follows a modular structure. The system is accessed via a web interface where the user can enter the target domain name or IP address to be localized. PlanetLab landmarks measure delays to the target and the results are forwarded to the evaluation module. This module applies the proposed probabilistic model to determine the location of the requested IP address. After evaluation, the expected target position is returned to the user, while the individual cell probabilities are visualized on Google Maps. Both the measurement data and the resulting cell probabilities are stored in our database. The following subsections highlight the key components of the system.

1) Data Collection: Spotter measures 10 round-trip delays from each PlanetLab landmark to the target node. The evaluation module extracts the base values, i.e. the minimal roundtrip delay for each landmark. We assume that this way the effect of queuing can be significantly decreased.

2) Model Evaluation: In its default setting Spotter uses the probabilistic model described in Section III and IV. To determine the location estimates as described in Section III we need to divide the surface of the globe into finite number of regions. For this step, we use the Hierarchical Triangular Mesh 


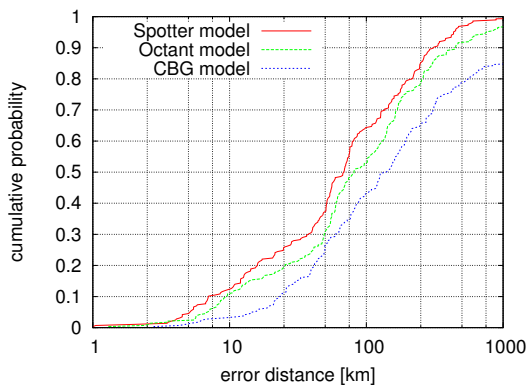

(a) PLANETLAB in North America

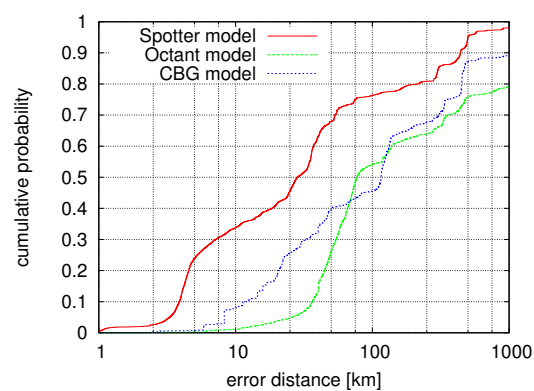

(d) COGENT in North America

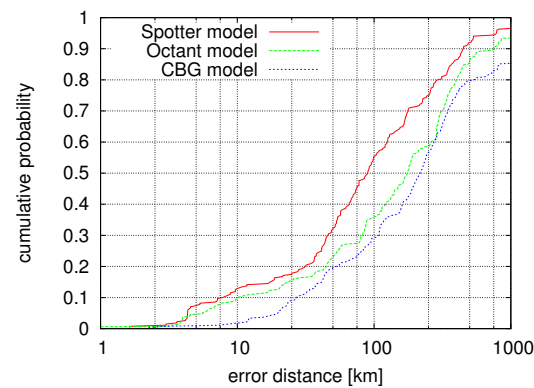

(b) PLANETLAB in Europe

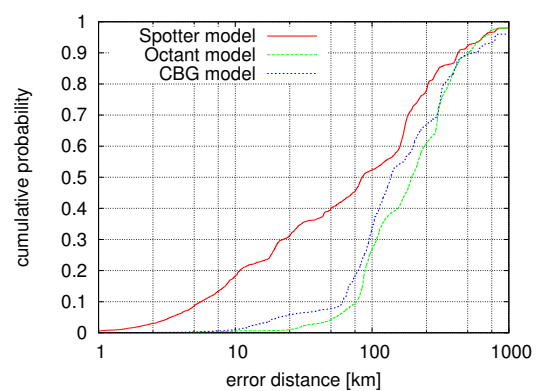

(e) COGENT in Europe

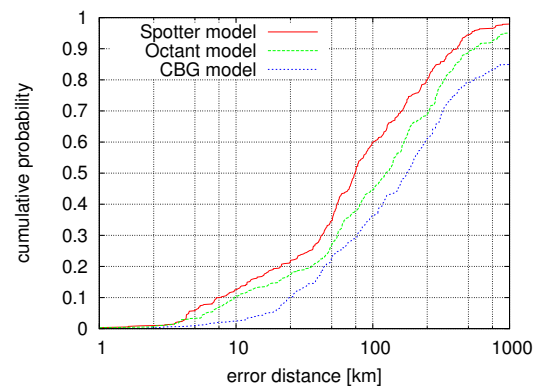

(c) PLANETLAB over the world

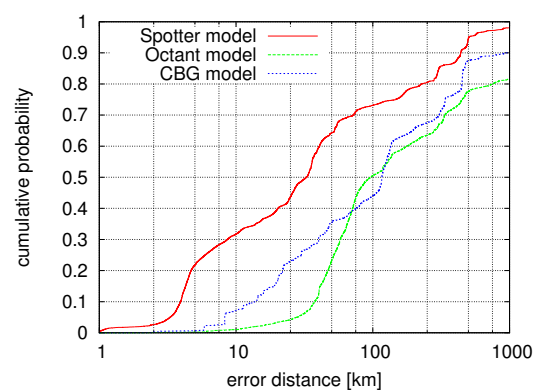

(f) COGENT over the world

Figure 4. Accuracy of the underlying models of Spotter, CBG and Octant on the PLANETLAB and COGENT reference data sets. The upper row contains the CDF plots of the localization errors for the three methods evaluated on PLANETLAB reference data, while the figures in the lower row show the error distributions on the more realistic COGENT ground truth collected by CAIDA. From left to right the columns represent these error distributions separately for three geographic regions: North America, Europe, and all over the world respectively.

(HTM) [16] which is a multi level, recursive decomposition of the sphere. It subdivides the surface into spherical triangles of similar shapes and sizes. This method is based on a quad-tree that is particularly good at supporting searches at different resolutions from arc seconds to hemispheres. At a given resolution level Spotter determines the probability value for each HTM cell by approximating the integral in (2). If required the results can be combined with other probability surfaces such as those derived from population densities, city locations or other geographic constraints.

The HTM library [17] provides very good performance in calculating spatial quantities such as spherical distance, area, intersecting regions, etc, and thus helps making the Spotter implementation efficient. In addition, the hierarchical structure of HTM provides flexibility in choosing the cell size for the calculations. Therefore the trade-off between running time and geographic resolution can be conveniently fine tuned.

3) Visualization: The outcome of the evaluation is a spatial probability distribution and its moments. These results can be visualized on geographic maps (for examples, see Section VI). The Spotter web interface displays the estimated region and the expected location on a Google Maps application.

4) Data Handling: The whole process is supported by the Network Measurement Virtual Observatory [18] as its backend database engine. Both the raw delay data and the localization results are kept in this database and can be accessed publicly.

In the Spotter implementation the applied delay-distance model is not hard coded into the evaluation module, instead it is precalculated and stored in the database. Due to the common database representation of models Spotter can easily work with different delay-distance models. This feature is beneficial for comparing and enhancing geolocation approaches.

\section{B. The Web Interface}

The Spotter service sits on top of the workflow presented previously and is available at [5]. It supports two localization modes. The single target mode expects an IP address or domain name as input for localization. After submitting a request the system geolocates the address and displays the results. This mode is open for everybody to submit queries that are performed immediately. Since the execution of the workflow takes nearly a minute in this mode, it is not suitable for larger node lists.

To optimize the localization process for multiple targets the batch target mode can be used. Due to both security and performance considerations the batch mode requires an initial registration, after which users can upload a text file containing the target addresses. Upon the targets in the list are localized, Spotter sends the results back to the user by email.

\section{Scalability}

As Spotter is intended to localize large IP sets, scalability becomes a key issue. We investigate Spotter's performance by running the data collection and evaluation processes on target lists with different sizes. We use the COGENT reference IP set described in Section IV-C to create the benchmark target lists. From the 23000 original addresses we created 20 randomly selected target lists of size 1000, 2000, ..., 20000. These lists 
were passed to the $\mathrm{C \#}$ implementation of Spotter running on a Dell Server with two Intel Xeon $2.5 \mathrm{GHz}$ CPUs and 32 GB Memory. For each list we recorded the time needed for performing round-trip delay measurements and the running time of the evaluation process. The benchmark procedure was repeated 10 times on different randomly sampled address lists to obtain average running times for each list size. The benchmark scenario shows that the data collection phase takes significantly larger share from the overall running time since we use low packet probe rates to avoid network flooding, e.g. for 10000 distinct targets preforming the delay measurements takes 25 minutes while the model evaluation phase is 7 minutes long. Additionally, the localization takes a few minutes even for very small target lists, due to the overhead of the communication with the PlanetLab nodes. Both the data collection and the model evaluation show linear connection with the number of targets, indicating that Spotter enables the localization of large IP sets in reasonable time.

\section{LARge Scale Application Scenarios}

To demonstrate the power of Spotter for large scale IP geolocalization we give a brief insight into two possible experimental scenarios. First, we determine the geographic position of YouTube video streaming servers [19] for which the existing services return false positions. Second, we illustrate that a reliable localization service can be beneficial for network topology visualization and opens the door to analyze its geographical properties. Since our methodology can be used to determine the spatial probability distribution for each target, by aggregating these individual spatial distributions we can derive the joint probability map of multiple geographically dispersed target hosts. This joint distribution describes the possible locations of the whole IP set together and can be applied for geographical visualization of large IP domains.

\section{A. YouTube Server Locations}

YouTube is a large scale content provider system controlling thousands of data storages all over the world. In the sense of Quality of Service their performance is extremely affected by the geographic distance of the servers and the user. These data centers are the most popular targets among the Internet users and generate a significant proportion of the whole network traffic. This is the reason why ISPs are highly interested in their topological and geographical aspects.

In this study we focus on IP addresses belonging to the 74.125.0.0/16 address block serving most of the YouTube contents. As we discussed in Section II the commercial solutions like MaxMind locate mistakenly all these addresses in Mountain View, CA. Next, we show that with Spotter the localization of these targets is feasible.

As a first step we identified 9312 active servers (responding to ICMP requests) out of the 65536 total addresses. Then, these hosts were localized by Spotter using the previously described batch mode. The localization results are presented in Figures 5(a)-5(c). The maps show the aggregated probability density of the possible locations of YouTube servers. The places with large densities represent the largest data centers. One can observe that in North America 8 centers can be identified. The largest ones are located in Atlanta, Seattle, Chicago and San Francisco. In Europe there are only 3 large sites hosting YouTube contents: Frankfurt, Amsterdam and London. In Asia 5 different locations can be found, in Tokyo, Osaka, Taipei, Hong Kong and Singapore. The next use case shows that the locations of these content delivery centers are in accordance with the largest Internet junctions traversed by the significant fraction of end-to-end network paths.

\section{B. PlanetLab Router Map}

In the last decade the structure of the Internet topology has been studied from many aspects [20], [21], but only a few work deals with the geographical properties of network elements [6], [22]. Since Spotter is a publicly available service it can be used to discover and analyze these hidden attributes. In this section we overview a router localization scenario which is fundamental for examining the geographical properties of the Internet. In order to cover a dispersed part of the Internet we utilized more than 700 PlanetLab nodes across all over the world. For discovering IP addresses along the paths between all the PlanetLab node pairs we performed Paris traceroute measurements [20]. From the collected more than 400 thousand traceroute experiments we identified 15725 distinct IP addresses. Note, that as different IP addresses might belong to unique interfaces of the same router the real number of individual routers might be less.

Similarly to the previous scenario the Spotter's batch localization mode was applied to determine the spatial density map for the network entities. The aggregated probability density maps of the inter-PlanetLab routers can be seen on Figures 5(d)-5(f). These routers are geographically much more dispersed than the YouTube servers. By comparing the two figure sets, one can recognize that the YouTube sites are deployed in cities which denote frequent places on the router maps, such as San Francisco, Frankfurt or Tokyo. The detailed analysis of these results are presented in [22].

\section{CONCLUSION}

In this paper we have proposed a probabilistic method and an online service built on the top of it which estimates the geographic location of Internet devices with $30 \mathrm{~km}$ median precision. Our approach uses a statistical description of the relationship between network delay and geographical distance. While the existing methods are based on the presumption that the delay-distance relation carries landmark specific characteristics this study shows that there exists generic statistical description of delay-distance behavior. According to this fundamental discovery, Spotter does not require calibration for each individual landmark but derives a common delay-distance model. To demonstrate the robustness and the accuracy of Spotter we test the performance on both PlanetLab nodes as well as on the novel COGENT reference set collected by CAIDA. On both data sets our probabilistic model outperforms the state-of-the-art models of CBG and Octant. This paper 


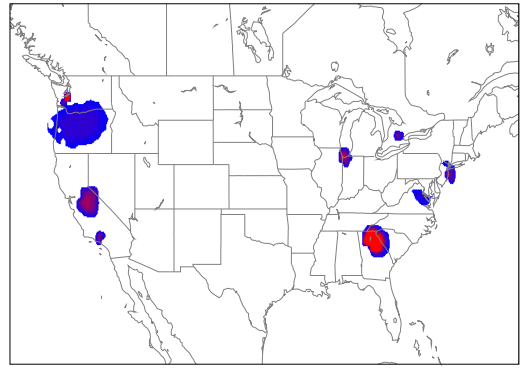

(a) YouTube sites in North America

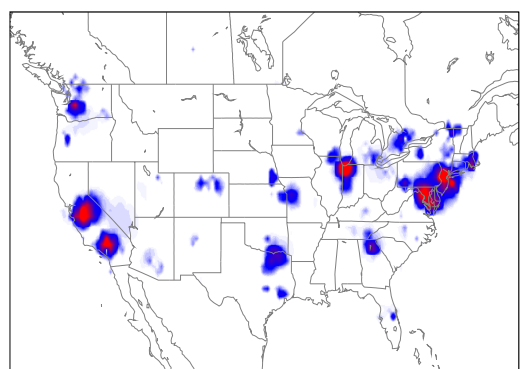

(d) Inter-PlanetLab routers in North America

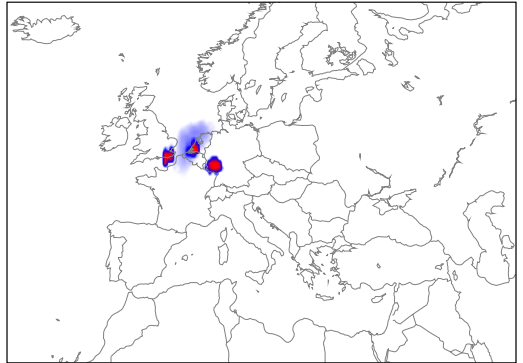

(b) YouTube sites in Europe

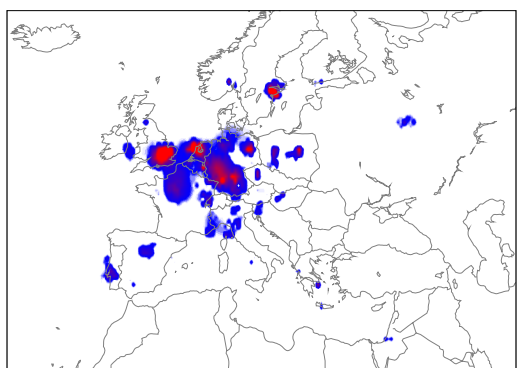

(e) Inter-PlanetLab routers in Europe

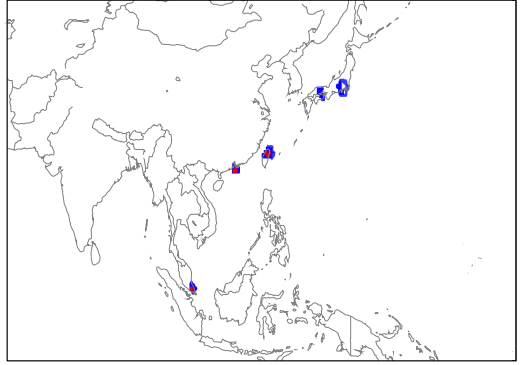

(c) YouTube sites in Asia

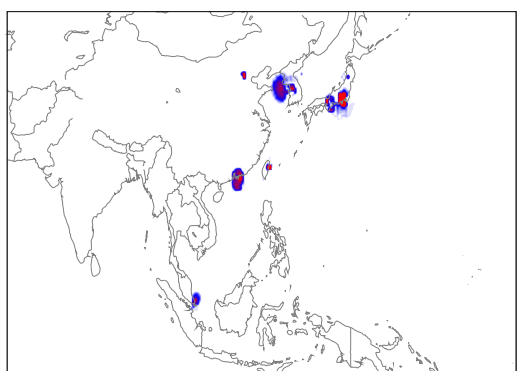

(f) Inter-PlanetLab routers in Asia

Figure 5. Two localization scenarios for demonstrating the power of Spotter. The density maps in the upper row shows the geographical distribution of the IP domain serving most of the YouTube contents, while the maps in the lower row represent the possible locations of routers along network paths between all the PlanetLab node pairs.

shows that the Spotter's underlying probabilistic model is less prone to measurement errors and other anomalies than the prior techniques.

To manifest the robustness and applicability of our approach we developed an online geolocation service that gives an effective way for positioning large number of IP addresses. We believe that Spotter will stimulate the research in the field by providing a valuable tool for understanding the geographical aspects of Internet.

\section{ACKNOWLEDGEMENTS}

We would like to thank Bradley Huffaker from CAIDA and Cogent for providing us the reference dataset. This work was partially supported by the National Office for Research and Technology (NAP 2005/ KCKHA005), the National Science Foundation OTKA 7779 and 80177, the National Development Agency (TAMOP 4.2.1/B-09/1/KMR-2010-0003), the EU ICT OneLab2 (Grant No.224263) and the EU FIRE NOVI project (Grant No.257867).

\section{REFERENCES}

[1] S. Laki, P. Mátray, P. Hága, I. Csabai, G. Vattay: "A Model Based Approach for Improving Router Geolocation", Computer Networks, Volume 54, Issue 9 , 17 June 2010, Pages 1490-1501, (2010).

[2] Bamba Gueye, Artur Ziviani, Mark Crovella and Serge Fdida "ConstraintBased Geolocation of Internet Hosts", in IEEE/ACM Transactions on Networking, 14(6):1219-1232.

[3] B. Wong, I. Stoyanov, E.G. Sirer: "Octant: A Comprehensive Framework for the Geolocalization of Internet Hosts", NSDI 2007 Symposium, Cambridge, Massachusetts, April 2007.

[4] CAIDA - The Cooperative Association for Internet Data Analysis

[5] The SPOTTER website:, http://spotter.etomic.org
[6] Venkata N. Padmanabhan, Lakshminarayanan Subramanian, "An investigation of geographic mapping techniques for internet hosts", Proceedings of ACM SIGCOMM, p.173-185, August 2001, San Diego, CA, USA.

[7] M. Zhang, Y. Ruan, V. Pai, and J. Rexford, "How DNS misnaming distorts Internet topology mapping", USENIX Conference, 2006.

[8] Bamba Gueye, Steve Uhlig and Serge Fdida, "Investigating the Imprecision of IP Block-Based Geolocation", PAM 2007, Louvain-la-neuve, Belgium, LNCS 4427, pp. 237-240, 2007

[9] E. Katz-Bassett, J. John, A. Krishnamurthy, D. Wetherall, T. Anderson, Y. Chawathe: "Towards IP Geolocation using Delay and Topology Measurements", ACM IMC 2006, p71-84, Rio de Janeriro, Brazil (2006).

[10] Y. Shavitt and N. Zilberman, "A study of geolocation databases", arXiv: $1005.5674,2010$

[11] MaxMind, http://www.maxmind.com

[12] Digital Envoy NetAcuity, http://www.digital-element.com

[13] Quova, http://www.quova.com

[14] M. Anderson, A. Bansal, B. Doctor, G. Hadjiyiannis, C. Herringshaw, E. Karplus and D. Muniz: "Method and apparatus for estimating a geographic location of a networked entity" United States Patent 6,684,250. Assigned to Quova, Inc. Filed 3 April 2001. Issued 27 January 2004.

[15] Brian Eriksson, Paul Barford, Joel Sommersy, and Robert Nowak: "A Learning-based Approach for IP Geolocation"', PAM 2010, April, 2010.

[16] Alex Szalay, Jim Gray, Gyorgy Fekete, Peter Kunszt, Peter Kukol, and Ani Thakar, "Indexing the Sphere with the Hierarchical Triangular Mesh", Technical Report, MSR-TR-2005-123, Microsoft Research, 2005

[17] The HTM library, http://skyserver.org/htm

[18] P. Mátray, I. Csabai, P. Hága, J. Stéger, L. Dobos, G. Vattay: "Building a Prototype for Network Measurement Virtual Observatory", ACM SIGMETRICS - MineNet 2007, 12 June 2007, San Diego, CA, USA

[19] YouTube, http://www.youtube.com

[20] B. Augustin, X. Cuvellier, B. Orgogozo, F. Viger, T. Friedman, M. Latapy, C. Magnien and R. Teixeira, "Avoiding traceroute anomalies with Paris traceroute", IMC 2006, October 2006.

[21] Y. Shavitt, Y. Singer, "Limitations and Possibilities of Path Trading Between Autonomous Systems", IEEE INFOCOM 2010, March 2010 , San Diego, CA, USA.

[22] P. Mátray, P. Hága, S. Laki, I. Csabai, G. Vattay, "On the Network Geography of the Internet", IEEE INFOCOM 2011, April 2011, Shanghai, China 